\title{
A MULTILEVEL DISCONTINUOUS GALERKIN METHOD
}

\author{
J. GOPALAKRISHNAN AND G. KANSCHAT
}

\begin{abstract}
A variable V-cycle preconditioner for an interior penalty finite element discretization for elliptic problems is presented. An analysis under a mild regularity assumption shows that the preconditioner is uniform. The interior penalty method is then combined with a discontinuous Galerkin scheme to arrive at a discretization scheme for an advection-diffusion problem, for which an error estimate is proved. A multigrid algorithm for this method is presented, and numerical experiments indicating its robustness with respect to diffusion coefficient are reported.
\end{abstract}

\section{INTRODUCTION}

In this paper we show that a multigrid technique can be used for efficient solution of linear systems arising from the so-called interior penalty finite element method for second order elliptic boundary value problems. We also present a fast method for advection-diffusion equations by combining the interior penalty method with the discontinuous Galerkin method for transport equations, and applying a multigrid technique on the resulting discrete system.

Discontinuous Galerkin (DG) methods have traditionally been used in numerical solution of hyperbolic conservation laws [12, 20, 22]. Their ability to capture strong gradients in solution without spurious oscillations is well known. Recently DG methods have also been shown to be of use in solving elliptic problems [11]. It is now common to classify various earlier methods for solving elliptic problems that went by the name of "interior penalty methods" $[2,3,13,27]$ under DG methods (see [1] for a unified treatment). In general, interior penalty finite element methods use discontinuous finite element functions, but penalize discontinuities of the function or its derivatives across inter-element boundaries.

Part of this paper deals with the interior penalty method considered in $[2,27]$. When applied to elliptic problems, the method gives rise to linear systems with condition number that grows like $O\left(h^{-2}\right)$ on quasi-uniform grids with mesh size $h$. We prove (in Section 3) under weak regularity

1991 Mathematics Subject Classification. 65F10, 65N55, 65N30.

Key words and phrases. discontinuous Galerkin, interior penalty, finite element method, multigrid, V-cycle, preconditioning, advection-diffusion.

This research was supported in part by Medtronic Inc., Supercomputing Institute of University of Minnesota, and Deutsche Forschungsgemeinschaft. 
assumptions that if a variable $\mathrm{V}$-cycle multigrid operator is used to precondition the linear system, then the resulting condition number is $O(1)$, i.e., bounded independently of $h$. The proof is an application of the abstract multigrid theory of [8] for non-inherited bilinear forms. The conjugate gradient method using this preconditioner converges in $O(N)$ operations, where $N$ is the number of unknowns, thus yielding an asymptotically optimal solution technique. In Section 4, we confirm and illustrate the theoretical result through numerical experiments.

DG schemes show their full potential in advection problems rather than elliptic problems. In Section 5, we introduce a DG scheme for an advectiondiffusion equation with an arbitrarily small diffusion term. This scheme reduces to the standard DG method for advection problems when the diffusion term is zero. On the other hand, when the advection term is zero, our scheme is the interior penalty method. We prove error estimates that show that the method yields good approximate solutions. An error analysis for a somewhat similar (but nonsymmetric) method has been reported recently [18]. Nonetheless, our proof (in Section 6) yields an error estimate in a slightly stronger norm.

For this scheme, we also present a computational technique for fast solution that performs uniformly in both convection dominated and diffusion dominated regimes. This is inspired by the fact that a downwind GaußSeidel iteration is an exact solver in the case of zero diffusion. Although this is no longer true when the diffusion term is nonzero, in this case, as shown in Sections 3 and 4, multigrid works well. Therefore, we investigate the performance of a multigrid method with the downwind Gauß-Seidel iteration as smoother in Section 5. Similar ideas have appeared earlier [9, 17], although these are in connection with other discretization schemes. Two multigrid analyses for advection-diffusion equations can be found in [23, 24]. Both works deal with the streamline diffusion method and it is not clear if the properties of a downwind ordering can be exploited in these analyses.

\section{Multilevel SPACES AND THe INTERior PENALty Method}

In this section we introduce notation for multilevel spaces and describe an interior penalty method. Before we define discrete spaces associated with the interior penalty method of $[2,27]$, we describe a model problem and state a mild regularity assumption on its solutions.

Let us first define Sobolev spaces that we will use. For a bounded connected open subset $\mathcal{D}$ of $\mathbb{R}^{2}$ or $\mathbb{R}$, let $L^{2}(\mathcal{D})$ denote the space of square integrable functions on $\mathcal{D}$, and let $(\cdot, \cdot)_{\mathcal{D}}$ and $\|\cdot\|_{0, \mathcal{D}}$ denote the innerproduct and norm on $L^{2}(\mathcal{D})$ (or $L^{2}(\mathcal{D})^{2}$ ) respectively. Denote by $H_{0}^{1}(\mathcal{D})$ the completion of compactly supported infinitely differentiable functions under the norm

$$
|u|_{1, \mathcal{D}} \equiv\|\nabla u\|_{0, \mathcal{D}}
$$


Let the dual space of $H_{0}^{1}(\mathcal{D})$ be denoted by $H^{-1}(\mathcal{D})$. For $-1<s<0$, let $H^{-s}(\mathcal{D})$ denote the the space obtained by interpolation (by the real number method) between $H^{-1}(\mathcal{D})$ and $L^{2}(\mathcal{D})$. For non-negative integers $m$, the Sobolev space $H^{m}(\mathcal{D})$ is the set of functions in $L^{2}(\mathcal{D})$ with distributional derivatives up to order $m$ also in $L^{2}(\mathcal{D})$. If $s$ is a positive real number between non-negative integers $m$ and $m+1, H^{s}(\mathcal{D})$ is the space obtained by interpolation between $H^{m}(\mathcal{D})$ and $H^{m+1}(\mathcal{D})$. The norm on $H^{r}(\mathcal{D})$ for any $r$ is denoted by $\|\cdot\|_{r, \mathcal{D}}$.

For transparent presentation of multigrid analysis we will restrict ourselves to the following simple model problem: Find $U \in H_{0}^{1}(\Omega)$ such that

$$
(\nabla U, \nabla \phi)_{\Omega}=(f, \phi)_{\Omega} \quad \text { for all } \phi \in H_{0}^{1}(\Omega),
$$

where $\Omega \subset \mathbb{R}^{2}$ is a polygonal domain, $\partial \Omega$ denotes the boundary of $\Omega$, and $f \in L^{2}(\Omega)$. We will make the following regularity assumption on solutions to this problem: There is an $1 / 2<\alpha \leq 1$ and a constant $C_{\Omega}$ such that the solution $U$ of (2.1) satisfies

$$
\|U\|_{1+\alpha, \Omega} \leq C_{\Omega}\|f\|_{-1+\alpha, \Omega} .
$$

This assumption is known to hold [21] for polygonal domains with $\alpha<\delta^{-1}$ where $\pi \delta$ is the maximum of the interior angles of $\Omega$. For convex domains, it holds [15] with $\alpha=1$.

Let $\Omega$ be subdivided by a "coarse" quasiuniform triangulation $\mathcal{T}_{1}$ of meshsize $h_{1}$. Our multilevel spaces are based on a sequence of refinements of this mesh. We refine the triangulation $\mathcal{T}_{1}$ to produce $\mathcal{T}_{2}$ by splitting each triangle of $\mathcal{T}_{1}$ into four congruent triangles. The triangulation $\mathcal{T}_{2}$ is then quasiuniform with mesh-size $h_{2}=h_{1} / 2$. Repeating this process, we get a sequence of triangulations $\mathcal{T}_{k}, k=1, \ldots J$, each quasiuniform with meshsize $h_{k}=h_{1} / 2^{k-1}$. Let $d$ be a fixed integer not less than one. We define multilevel spaces $M_{1} \subset M_{2} \subset \ldots M_{J}$ by

$$
M_{k}=\left\{v:\left.v\right|_{\tau} \text { is a polynomial of degree at most } d, \text { for all } \tau \in \mathcal{T}_{k}\right\} .
$$

We can also let $\mathcal{T}_{k}$ to be a mesh with quadrilateral elements and $M_{k}$ to be a mapped tensor-product finite element space. Let us now describe the interior penalty method at each refinement level in terms of these spaces.

The interior penalty method provides a discontinuous Galerkin approximation to the solution $U$ of (2.1). To describe it, we will need the spaces

$$
H^{1}\left(\mathcal{T}_{k}\right)=\left\{u \in L^{2}(\Omega):\left.u\right|_{\tau} \in H^{1}(\tau) \text { for all } \tau \in \mathcal{T}_{k}\right\}
$$

Let $\varepsilon_{k}$ denote the set of edges of the triangulation $\mathcal{T}_{k}$. If $e \in \mathcal{E}_{k}$ is an interior edge, denoting by $n_{e}$ one of the two unit normal vectors at $e$, we define jumps and averages of normal derivatives (for $x \in e$ ) of $u \in H^{1}\left(\mathcal{T}_{k}\right.$ ) by

$$
\begin{aligned}
{[u]_{e}(x) } & =\lim _{\delta \rightarrow 0+}\left(u\left(x-\delta n_{e}\right)-u\left(x+\delta n_{e}\right)\right), \quad \text { and } \\
\left\langle\partial_{n} u\right\rangle_{e}(x) & =\frac{1}{2} \lim _{\delta \rightarrow 0+}\left(n_{e} \cdot \nabla u\left(x-\delta n_{e}\right)+n_{e} \cdot \nabla u\left(x+\delta n_{e}\right)\right),
\end{aligned}
$$


while if $e \subseteq \partial \Omega$, we fix $n_{e}$ to be the outward normal vector and let

$$
[u]_{e}(x)=\lim _{\delta \rightarrow 0+} u\left(x-\delta n_{e}\right), \quad \text { and } \quad\left\langle\partial_{n} u\right\rangle_{e}=\lim _{\delta \rightarrow 0+} n_{e} \cdot \nabla u\left(x-\delta n_{e}\right) .
$$

Here and elsewhere, we use "." to denote innerproducts in Euclidean spaces. We will drop the subscript $e$ when no confusion can arise. Define $a_{k}(\cdot, \cdot)$ on $H^{1}\left(\mathcal{T}_{k}\right) \times H^{1}\left(\mathcal{T}_{k}\right)$ by

$a_{k}(u, v)=\sum_{\tau \in \mathcal{T}_{k}}(\nabla u, \nabla v)_{\tau}+\sum_{e \in \mathcal{E}_{k}}\left(\frac{\sigma}{\ell_{e}}([u],[v])_{e}-\left(\left\langle\partial_{n} u\right\rangle,[v]\right)_{e}-\left([u],\left\langle\partial_{n} v\right\rangle\right)_{e}\right)$.

Here $\ell_{e}$ denotes the length of edge $e$, and $\sigma$ is a positive parameter to be chosen. The interior penalty method is based on the observation that the $U$ that solves the Poisson equation (2.1) also satisfies

$$
a_{k}(U, v)=(f, v)_{\Omega} \quad \text { for all } v \in H^{1}\left(\mathcal{T}_{k}\right),
$$

for each $k=1, \ldots J$. The interior penalty approximation to $U$ from $M_{k}$, namely $U_{k}$, is defined by

$$
a_{k}\left(U_{k}, v_{k}\right)=\left(f, v_{k}\right) \quad \text { for all } v_{k} \in M_{k} .
$$

Here and elsewhere, when the subscript indicating the domain in $L^{2}$ innerproduct is dropped, the domain is to be taken as $\Omega$.

If $\sigma$ is chosen large enough, the discrete system (2.5) is uniquely solvable [2]. This follows from the inverse inequality

$$
\sum_{e \in \mathcal{E}_{k}} \ell_{e}\left\|\left\langle\partial_{n} u\right\rangle\right\|_{0, e}^{2} \leq C_{0} \sum_{\tau \in \mathcal{T}_{k}}\|\nabla u\|_{0, \tau}^{2}
$$

which holds for all $u \in M_{k}$ and for all $k=1, \ldots J$, with the constant $C_{0}$ independent of $\left\{h_{k}\right\}$. Indeed, whenever $\sigma>2 C_{0}$, it can easily be seen that

$$
a_{k}(u, u) \geq \frac{1}{2} \sum_{\tau \in \mathcal{T}_{k}}\|\nabla u\|_{0, \tau}^{2}+\left(\sigma-2 C_{0}\right) \sum_{e \in \mathcal{E}_{k}} \frac{1}{\ell_{e}}\|[u]\|_{0, e}^{2},
$$

for all $u \in M_{k}$. Then $a_{k}(\cdot, \cdot)$ is a positive definite and symmetric bilinear form, and consequently is an innerproduct on $M_{k}$. Therefore (2.5) is uniquely solvable. The norm generated by $a_{k}(\cdot, \cdot)$ will be denoted by $\|\cdot\| \|_{k}$, i.e.,

$$
\|u\|_{k} \equiv a_{k}(u, u)^{1 / 2} .
$$

We will henceforth assume that $\sigma>2 C_{0}$. Freedom in choice of $\sigma$ in actual practice can be a boon [2] or bane depending on available information.

In [2], the interior penalty method is proved to yield good approximations to $U$ in a certain "energy norm", slightly different from $\|\cdot\|_{k}$. Nonetheless, it is easy to get estimates in $\|\cdot\|_{k}$-norm as well. Indeed, Galerkin orthogonality and the Cauchy-Schwarz inequality for $a_{k}(\cdot, \cdot)$ imply

$$
\left\|U-U_{k}\right\|_{k} \leq\left\|U-v_{k}\right\|_{k} \quad \text { for all } v_{k} \in M_{k} .
$$


In particular, we may choose for $v_{k}$ an interpolant of $U$ that is continuous on $\Omega$ and vanishes on $\partial \Omega$. Let $\mathcal{J}_{k}$ denote such an interpolation operator [26], having the property that

$$
\left|v-\mathcal{J}_{k} v\right|_{1, \Omega} \leq C h_{k}^{\alpha}\|v\|_{1+\alpha, \Omega} \quad \text { for all } v \in H^{1+\alpha}(\Omega) .
$$

We have adopted the usual convention of denoting by $C$ (with or without subscript) a generic constant independent of $h_{k}$. At any two different occurrences, its value may differ, but will always remain independent of mesh sizes. Since $\left\|U-\mathcal{J}_{k} U\right\|_{k}=\left|U-\mathcal{J}_{k} U\right|_{1, \Omega}$, we can choose $v_{k}=\mathcal{J}_{k} U$ in (2.9) and get

$$
\left\|U-U_{k}\right\|_{k} \leq C h_{k}^{\alpha}\|U\|_{1+\alpha, \Omega} .
$$

Our interest is in efficiently computing the interior penalty approximation on the finest level, namely $U_{J}$. Let us define the operator $A_{k}: M_{k} \rightarrow M_{k}$ by

$$
\left(A_{k} u, v\right)=a_{k}(u, v) \quad \text { for all } u, v \in M_{k},
$$

and examine its spectral properties. Let $\lambda_{k}$ denote the maximum eigenvalue of $A_{k}$, i.e.,

$$
\lambda_{k}=\sup _{v \in M_{k}} \frac{a_{k}(v, v)}{(v, v)} .
$$

From the trace inequality

$$
C \sum_{e \in \mathcal{E}_{k}} \frac{1}{\ell_{e}}\|[v]\|_{0, e}^{2} \leq \sum_{\tau \in \mathcal{T}_{k}} h_{k}^{-2}\|v\|_{0, \tau}^{2}+|v|_{1, \tau}^{2}, \quad \text { for all } v \in H^{1}\left(\mathcal{T}_{k}\right),
$$

the inverse inequality

$$
\sum_{\tau \in \mathcal{T}_{k}}\|\nabla v\|_{0, \tau}^{2} \leq C h_{k}^{-2}\|v\|_{0, \Omega}^{2}, \quad \text { for all } v \in M_{k},
$$

and (2.6), we get that

$$
\lambda_{k} \leq C\left(\sigma+C_{0}\right) h_{k}^{-2} .
$$

Remark 2.1. The minimum eigenvalue of $A_{k}$ is bounded from below by a constant independent of $h_{k}$. To see this, note that (2.7) implies

$$
\begin{gathered}
a_{k}(u, u) \geq C \min \left(1 / 2, \sigma-2 C_{0}\right) \tilde{a}_{k}(u, u), \quad \text { where } \\
\widetilde{a}_{k}(u, u)=\sum_{\tau \in \mathcal{T}_{k}}\|\nabla u\|_{0, \tau}^{2}+\sum_{e \in \mathcal{E}_{k}} \frac{1}{\ell_{e}}\|[u]\|_{0, e}^{2},
\end{gathered}
$$

for all $u \in M_{k}$. The assertion on minimum eigenvalue will follow if we show that

$$
\widetilde{a}_{k}(u, u) \geq C\|u\|_{0, \Omega}^{2}
$$

This is proved for convex domains in [2, Lemma 2.1]. Let us show here that this holds for any polygonal domain using [25, Theorem 3.1]. Let $V_{k}$ be the space of vector functions corresponding to $M_{k}$ such that $\left(V_{k}, M_{k}\right)$ form a Raviart-Thomas pair of spaces [10]. Also let $\nabla_{k}: M_{k} \mapsto V_{k}$ be the 
$L^{2}$-adjoint of the divergence map div $: V_{k} \mapsto M_{k}$. Then [25, Theorem 3.1] states that $\widetilde{a}_{k}(u, u) \geq C\left\|\nabla_{k} u\right\|_{0, \Omega}^{2}$. We also have that

$$
\left\|\nabla_{k} u\right\|_{0, \Omega} \geq \sup _{q \in V_{k}} \frac{\left(\nabla_{k} u, q\right)}{\|q\|_{0, \Omega}} \geq \sup _{q \in V_{k}} \frac{\left(\nabla_{k} u, q\right)}{\|q\|_{H(\mathrm{div})}} \geq C\|u\|_{0, \Omega},
$$

where $\|q\|_{H(\text { div })}=\left(\|q\|_{0, \Omega}^{2}+\|\operatorname{div} q\|_{0, \Omega}^{2}\right)^{1 / 2}$, and the last inequality was because of the inf-sup condition for the pair $\left(V_{k}, M_{k}\right)$. Thus, (2.16) holds.

From (2.14) and Remark 2.1, we see that the condition number of the discrete system (2.5) is bounded by $C h^{-2}$. This estimate is sharp. Therefore, iterative solution of (2.5) for large problems calls for a good preconditioner. In the next section, we show that a multigrid technique can be used to precondition (2.5) effectively.

Remark 2.2. It is not difficult to show that $a_{k}(u, u) \leq C \sigma \widetilde{a}_{k}(u, u)$. This, together with (2.15), imply that any preconditioner for the form $\widetilde{a}_{k}(u, u)$ also gives a preconditioner for $a_{k}(u, u)$. In [25], an overlapping Schwarz preconditioner for $\widetilde{a}_{k}(u, u)$ is analyzed. This preconditioner can very well be used to precondition our discrete system (2.5).

\section{A VARIABle V-CyCle PRECONDItioner}

In this section, we define and analyze a variable V-cycle operator. Our main theorem will show that this operator provides a uniform preconditioner for (2.5). The multigrid analysis we present here is an application of the abstract theory of multigrid algorithms in $[6,8]$.

Let us first write (2.5) in matrix form. Let $N_{k}$ be the number of degrees of freedom of $M_{k}$. Let $\left\{\phi_{k}^{i}\right\}_{i=1}^{N_{k}}$ be the nodal basis for $M_{k}$. Let $\mathrm{A}_{k}$ be the matrix whose $(i, j)$-th entry is $a_{k}\left(\phi_{k}^{j}, \phi_{k}^{i}\right)$. If $\mathrm{u}_{J}$ is the vector of coefficients in the expansion of $U_{J}$ in the nodal basis, then (2.5) implies that

$$
\mathrm{A}_{J} \mathrm{u}_{J}=\mathrm{b}_{J}
$$

where $\mathrm{b}_{J}$ is a vector whose $i$-th component equals $\left(f, \phi_{J}^{i}\right)$. We are interested in efficiently solving this equation. Our intention is to define a matrix $B_{J}$ such that $\mathrm{B}_{J} \mathrm{~A}_{J}$ is well conditioned and solve $\mathrm{B}_{J} \mathrm{~A}_{J} \mathrm{u}_{J}=\mathrm{B}_{J} \mathrm{~b}_{J}$ instead.

A main ingredient of multigrid algorithms are "smoother" matrices, which we denote by $\mathrm{R}_{k}$. Let $\mathrm{L}_{k}, \mathrm{U}_{k}$, and $\mathrm{D}_{k}$ be strictly lower triangular, strictly upper triangular, and diagonal matrices respectively such that $\mathrm{A}_{k}=\mathrm{L}_{k}+$ $\mathrm{D}_{k}+\mathrm{U}_{k}$. Define $\mathrm{J}_{k}$ and $\mathrm{G}_{k}$ by

$$
\mathrm{J}_{k}=\gamma \mathrm{D}_{k}^{-1} \quad \text { and } \quad \mathrm{G}_{k}=\left(\mathrm{D}_{k}+\mathrm{L}_{k}\right)^{-1},
$$

where $\gamma$ is an appropriate scaling factor. We will set $R_{k}$ to equal either $J_{k}$ or $\mathrm{G}_{k}$. Then the iteration

$$
\mathrm{x}^{i+1}=\mathrm{x}^{i}+\mathrm{R}_{k}\left(\mathrm{~b}_{k}-\mathrm{A}_{k} \mathrm{x}^{i}\right), \quad i=1,2, \ldots
$$

with some initial guess $x^{0}$, is either a scaled Jacobi or a Gauß-Seidel iteration, and both are well known [16] to be smoothing iterations. 
Multigrid algorithms also require intergrid transfer operators, often called "prolongation" and "restriction". These are given naturally in our application by the imbeddings $M_{1} \subset M_{2} \ldots \subset M_{J}$. Because of these imbeddings, there are numbers $\left\{\alpha_{i j}^{k}\right\}$ such that

$$
\phi_{k-1}^{i}=\sum_{i=1}^{N_{k}} \alpha_{i j}^{k} \phi_{k}^{i} .
$$

Let $C_{k-1}$ be a matrix whose $(i, j)$-th entry is the $\alpha_{i j}^{k}$ above. Then $C_{k-1}$ and its transpose $C_{k-1}^{t}$ are the restriction and prolongation matrices, respectively.

We can now define the variable $\mathrm{V}$-cycle matrix $\mathrm{B}_{J}$, following $[6,8]$. Instead of giving the entries of the matrix $\mathrm{B}_{J}$, the algorithm below defines $\mathrm{B}_{J}$ by defining its action on any vector. Thus, $\mathrm{B}_{J}$ (which is a full matrix) need not be assembled in an implementation. Let $m(k), k=1, \ldots, J$ be a sequence of positive integers.

Algorithm 3.1. Set $\mathrm{R}_{k}^{(l)}=\mathrm{R}_{k}$ if $l$ is odd, and $\mathrm{R}_{k}^{(l)}=\mathrm{R}_{k}^{t}$ if $l$ is even $\left(\mathrm{R}_{k}^{t}\right.$ is the transpose of $\mathrm{R}_{k}$ ). Also set $\mathrm{x}^{0}=0$ and $\mathrm{B}_{1}=\mathrm{A}_{1}^{-1}$. For $k \geq 2$ and any vector $\mathrm{d}_{k} \in \mathbb{R}^{N_{k}}, \mathrm{~B}_{k} \mathrm{~d}_{k}$ can be computed by the following four steps, assuming that $\mathrm{B}_{k-1}$ is already defined.

(1) Compute $\mathrm{x}^{l}$ for $l=1, \ldots m(k)$ :

$$
\mathrm{x}^{l}=\mathrm{x}^{l-1}+\mathrm{R}_{k}^{(l+m(k))}\left(\mathrm{d}_{k}-\mathrm{A}_{k} \mathrm{x}^{l-1}\right) .
$$

(2) Set $\mathrm{y}^{m(k)}=\mathrm{x}^{m(k)}+\mathrm{C}_{k-1}^{t} \mathrm{~B}_{k-1} \mathrm{C}_{k-1}\left(\mathrm{~d}_{k}-\mathrm{A}_{k} \mathrm{x}^{m(k)}\right)$.

(3) Compute $\mathrm{y}^{l}$ for $l=m(k)+1, \ldots, 2 m(k)$ :

$$
\mathrm{y}^{l}=\mathrm{y}^{l-1}+\mathrm{R}_{k}^{(l+m(k))}\left(\mathrm{d}_{k}-\mathrm{A}_{k} \mathrm{y}^{l-1}\right) .
$$

(4) Set $\mathrm{B}_{k} \mathrm{~d}_{k}=\mathrm{y}^{2 m(k)}$.

This algorithm thus defines $B_{J}$ recursively. We assume that the number of smoothings $m(k)$ increases as $k$ decreases in such a way that

$$
\beta_{0} m(k) \leq m(k-1) \leq \beta_{1} m(k),
$$

with $1<\beta_{0} \leq \beta_{1}$. A typical choice is $\beta_{0}=\beta_{1}=2$, i.e., $m(k)=2^{J-k}$. In this case the cost of the algorithm is comparable to that of the W-cycle. We can now give the main theorem.

Theorem 3.1. Suppose that the regularity assumption (2.2) holds and the number of smoothings satisfy (3.1). Then $\mathrm{B}_{J}$ is a symmetric and positive definite matrix and there is a constant $c_{\sigma}$ such that

$$
\zeta^{-1} \leq \frac{\left(\mathrm{B}_{J} \mathrm{~A}_{J} \mathrm{x}\right) \cdot\left(\mathrm{A}_{J} \mathrm{x}\right)}{\left(\mathrm{A}_{J} \mathrm{x}\right) \cdot \mathrm{x}} \leq \zeta, \quad \text { for all } \mathrm{x} \in \mathbb{R}^{N_{J}},
$$

with $\zeta=\left(c_{\sigma}+m(J)^{\alpha / 2}\right) / m(J)^{\alpha / 2}$. Here $c_{\sigma}$ is independent of mesh sizes $\left\{h_{k}\right\}$. Consequently, the spectral condition number of $\mathrm{B}_{J} \mathrm{~A}_{J}$, namely $\kappa\left(\mathrm{B}_{J} \mathrm{~A}_{J}\right)$, satisfies $\kappa\left(\mathrm{B}_{J} \mathrm{~A}_{J}\right) \leq \zeta^{2}$. 
From this theorem, it follows that $B_{J}$ is well suited for use in a preconditioned conjugate gradient iteration. Indeed, because of the bound on $\kappa\left(\mathrm{B}_{J} \mathrm{~A}_{J}\right)$, such an iteration will converge in a fixed number of steps independently of how many levels of refinement were used to obtain the fine mesh, i.e., independently of $h_{J}$. Note that even with one smoothing step at the finest level $(m(J)=1)$ we get a uniform preconditioner. Increasing $m(J)$ gives a better condition number estimate according to Theorem 3.1, but it also increases the cost of applying $\mathrm{B}_{J}$.

The proof of Theorem 3.1, by virtue of the abstract theory given in [8] (or see [6, Theorem 4.6]), reduces to verification of two conditions. Define $P_{k-1}: M_{k} \rightarrow M_{k-1}$ by

$$
a_{k-1}\left(P_{k-1} u, v_{k-1}\right)=a_{k}\left(u, v_{k-1}\right) \quad \text { for all } u \in M_{k}, v_{k-1} \in M_{k-1} \text {. }
$$

The two conditions are as follows:

(1) There exists an $0<\omega<2$ such that for all $k=2, \ldots, J$,

$$
\frac{\omega}{\lambda_{k}} \mathrm{M}_{k}^{-1} \mathrm{x} \cdot \mathrm{x} \leq 2 \mathrm{R}_{k} \mathrm{x} \cdot \mathrm{x}-\mathrm{A}_{k} \mathrm{R}_{k} \mathrm{x} \cdot \mathrm{R}_{k} \mathrm{x}, \quad \text { for all } \mathrm{x} \in \mathbb{R}^{N_{k}},
$$

where $\mathrm{M}_{k}$ is the mass matrix, i.e., its $(i, j)$-th entry is $\left(\phi_{k}^{i}, \phi_{k}^{j}\right)$.

(2) There is a $0<\beta \leq 1$ and $C_{P}>0$ such that

$$
\left|a_{k}\left(u-P_{k-1} u, u\right)\right| \leq C_{P}\left(\frac{\left\|A_{k} u\right\|_{k}^{2}}{\lambda_{k}}\right)^{\beta} a_{k}(u, u)^{1-\beta}
$$

holds for all $u \in M_{k}$, and for any $k=2, \ldots, J$.

The first condition pertains only to smoothers. In our application, this follows from the locality of basis functions. Indeed it can immediately be seen that the limited interaction and stable decomposition hypotheses in [7] hold in our case. It follows from [7, Theorem 3.2] that (3.3) holds for $\mathrm{R}_{k}=\mathrm{G}_{k}$ and from [7, Theorem 3.1] that there are values for $\gamma$ such that (3.3) holds for $\mathrm{R}_{k}=\mathrm{J}_{k}$. Note that the theory in [7] can also be used to conclude (3.3) for block Jacobi and Gauß-Seidel smoothers. Indeed these are the smoothers that we will use in our numerical experiments in later sections.

Therefore, to prove Theorem 3.1, it suffices to prove (3.4). In the remainder of this section, we will prove (3.4) with $\beta=\alpha / 2$, where $\alpha$ is as in the regularity assumption (2.2). The following lemma estimates the difference between $u$ and $P_{k-1} u$, and will be useful while proving Theorem 3.1.

Lemma 3.1. Assume that the regularity assumption (2.2) holds. Then, for all $u \in M_{k},(k=2, \ldots, J)$

$$
\left\|u-P_{k-1} u\right\|_{k} \leq C(\sigma) h_{k}^{\alpha}\left\|A_{k} u\right\|_{-1+\alpha, \Omega},
$$

where $C(\sigma)=C \sigma /\left(\sigma-2 C_{0}\right)$.

Proof. We start with

$$
\left\|u-P_{k-1} u\right\|_{k} \leq\|u-w\|_{k}+\left\|w-P_{k-1} u\right\|_{k},
$$


where $w \in H^{1+\alpha}(\Omega)$ satisfies

$$
-\Delta w=A_{k} u \quad \text { on } \Omega, \quad w=0 \quad \text { on } \partial \Omega .
$$

The proof now proceeds by estimating the two terms on the right hand side of (3.5).

From the consistency of the interior penalty method, we have that

$$
a_{k}(w, v)=\left(A_{k} u, v\right) \quad \text { for all } v \in H^{1}\left(\mathcal{T}_{k}\right)
$$

By the definition of $A_{k} u$, we also have that

$$
a_{k}\left(u, v_{k}\right)=\left(A_{k} u, v_{k}\right) \quad \text { for all } v_{k} \in M_{k} .
$$

Together, these equalities imply that $u$ is the interior penalty approximation to $w$ from $M_{k}$. Thus, by the error estimate (2.11),

$$
\|u-w\|_{k} \leq C h_{k}^{\alpha}\|w\|_{1+\alpha, \Omega} .
$$

It now remains only to estimate the last term in (3.5). As before, we have

$$
a_{k-1}(w, v)=\left(A_{k} u, v\right) \quad \text { for all } v \in H^{1}\left(\mathcal{T}_{k-1}\right) .
$$

In view of $(3.2)$, this equation implies that $P_{k-1} u$ is the interior penalty approximation of $w$ from $M_{k-1}$. Thus, by (2.11),

$$
\left\|w-P_{k-1} u\right\|_{k-1} \leq C h_{k-1}^{\alpha}\|w\|_{1+\alpha, \Omega} .
$$

We will now use (3.7) to estimate $\left\|w-P_{k-1} u\right\|_{k}$. Observe that $\left[w-P_{k-1} u\right]_{e}$ is zero for edges $e \in \mathcal{E}_{k}$ that are not subsets of a coarse edge in $\mathcal{E}_{k-1}$. Therefore,

$$
\sum_{e \in \mathcal{E}_{k}} \frac{1}{\ell_{e}}\left\|\left[w-P_{k-1} u\right]\right\|_{0, e}^{2}=\sum_{e \in \mathcal{E}_{k-1}} \frac{2}{\ell_{e}}\left\|\left[w-P_{k-1} u\right]\right\|_{0, e}^{2} .
$$

This implies that

$$
\left\|w-P_{k-1} u\right\|_{k}^{2} \leq\left\|w-P_{k-1} u\right\|_{k-1}^{2}+\sigma \sum_{e \in \mathcal{E}_{k-1}} \frac{1}{\ell_{e}}\left\|\left[w-P_{k-1} u\right]\right\|_{0, e}^{2} .
$$

Note that $[w]_{e}=0$ for all $e \in \mathcal{E}_{k-1}$. Consider the continuous interpolant $\mathcal{J}_{k-1} w$ of $w$. Then $\left[\mathcal{J}_{k-1} w\right]_{e}$ is zero as well. Hence,

$$
\begin{aligned}
\sigma \sum_{e \in \mathcal{E}_{k-1}} \frac{1}{\ell_{e}}\left\|\left[w-P_{k-1} u\right]\right\|_{0, e}^{2} & =\sigma \sum_{e \in \mathcal{E}_{k-1}} \frac{1}{\ell_{e}}\left\|\left[\mathcal{J}_{k-1} w-P_{k-1} u\right]\right\|_{0, e}^{2} \\
& \leq \frac{\sigma}{\sigma-2 C_{0}}\left\|\mathcal{J}_{k-1} w-P_{k-1} u\right\|_{k-1},
\end{aligned}
$$

where the last inequality was because of (2.7). Now since

$$
\left\|\mathcal{J}_{k-1} w-P_{k-1} u\right\|_{k-1} \leq\left\|w-\mathcal{J}_{k-1} w\right\|_{k-1}+\left\|w-P_{k-1} u\right\|_{k-1},
$$

and since $\left\|w-\mathcal{J}_{k-1} w\right\|_{k-1}=\left|w-\mathcal{J}_{k-1} w\right|_{1, \Omega},(2.10)$ and (2.11) imply that

$$
\sigma \sum_{e \in \mathcal{E}_{k-1}} \frac{1}{\ell_{e}}\left\|\left[w-P_{k-1} u\right]\right\|_{0, e}^{2} \leq C\left(\frac{\sigma}{\sigma-2 C_{0}}\right) h_{k-1}^{\alpha}\|w\|_{1+\alpha, \Omega} .
$$


Consequently, from (3.8) and (3.7), we get that

$$
\left\|w-P_{k-1} u\right\|_{k} \leq \frac{C \sigma}{\sigma-2 C_{0}} h_{k-1}^{\alpha}\|w\|_{1+\alpha, \Omega} .
$$

Combining this with (3.5), and using the regularity assumption (2.2) for $w$, we have the result.

Proof of Theorem 3.1. From the discussion before, it suffices to prove (3.4) with $\beta=\alpha / 2$. We will start with the result of Lemma 3.1:

$$
\left\|u-P_{k-1} u\right\|_{k} \leq C(\sigma) h_{k}^{\alpha}\left\|A_{k} u\right\|_{-1+\alpha, \Omega} \leq C(\sigma) h_{k}^{\alpha}\left\|A_{k} u\right\|_{-1, \Omega}^{1-\alpha}\left\|A_{k} u\right\|_{0, \Omega}^{\alpha},
$$

where the last inequality was because $H^{-1+\alpha}(\Omega)$ is in the scale of intermediate spaces between $L^{2}(\Omega)$ and $H^{-1}(\Omega)$. Let us now observe that if we show that

$$
\left\|A_{k} u\right\|_{-1, \Omega} \leq C\|u\|_{k},
$$

then (3.4) follows. Indeed, when (3.10) is combined with (3.11) and (2.14), we have that

$$
\begin{aligned}
a_{k}\left(u-P_{k-1} u, u\right) & \leq\left\|u-P_{k-1} u\right\|_{k}\|u\|_{k} \\
& \leq C(\sigma) h_{k}^{\alpha}\|u\|_{k}^{2-\alpha}\left\|A_{k} u\right\|_{0, \Omega}^{\alpha} \\
& \leq C(\sigma) \sigma^{\alpha / 2}\left(\frac{\left\|A_{k} u\right\|_{0, \Omega}^{2}}{\lambda_{k}}\right)^{\alpha / 2} a_{k}(u, u)^{1-\alpha / 2}
\end{aligned}
$$

from which (3.4) follows with $\beta=\alpha / 2$ and $C_{P}=C(\sigma) \sigma^{\alpha / 2}$.

Thus, it remains only to prove (3.11). For this we again use the continuous interpolant $\mathcal{J}_{k}$ of [26]. It is proved in [26] that for all $\psi \in H_{0}^{1}(\Omega)$,

$$
\begin{aligned}
\left|\mathcal{J}_{k} \psi\right|_{1, \Omega} & \leq C|\psi|_{1, \Omega} \\
\left\|\psi-\mathcal{J}_{k} \psi\right\|_{0, \Omega} & \leq C h_{k}|\psi|_{1, \Omega} .
\end{aligned}
$$

Now, to estimate $\left\|A_{k} u\right\|_{-1, \Omega}$, note that

$$
\begin{aligned}
\left\|A_{k} u\right\|_{-1, \Omega} & =\sup _{\psi \in H_{0}^{1}(\Omega)} \frac{\left(A_{k} u, \psi\right)}{|\psi|_{1, \Omega}} \\
& \leq \sup _{\psi \in H_{0}^{1}(\Omega)} \frac{\left(A_{k} u, \psi-\mathcal{J}_{k} \psi\right)}{|\psi|_{1, \Omega}}+\sup _{\psi \in H_{0}^{1}(\Omega)} \frac{a_{k}\left(u, \mathcal{J}_{k} \psi\right)}{|\psi|_{1, \Omega}} .
\end{aligned}
$$

Since $\left\|\mathcal{J}_{k} \psi\right\|_{k}=\left|\mathcal{J}_{k} \psi\right|_{1, \Omega}$, by (3.12) we have $\left\|\mathcal{J}_{k} \psi\right\|_{k} \leq C|\psi|_{1, \Omega}$. Using this, (3.13), and the Cauchy-Schwarz inequality, we get that

$$
\left\|A_{k} u\right\|_{-1, \Omega} \leq C h_{k}\left\|A_{k} u\right\|_{0, \Omega}+C\|u\|_{k} .
$$

Since $A_{k}$ is a symmetric positive definite operator, we also have

$$
\left\|A_{k} u\right\|_{0, \Omega}^{2} \leq \lambda_{k}\left(A_{k} u, u\right) \text {. }
$$

When this combined with (2.14) is used in (3.14), we get (3.11), thus completing the proof. 


\begin{tabular}{r|r|cc|cc} 
& & \multicolumn{2}{|c|}{$m(k)=2^{J-k}$} & \multicolumn{2}{c}{$m(k)=1$} \\
\cline { 3 - 6 }$J$ & $\kappa\left(\mathrm{A}_{J}\right)$ & $\kappa\left(\mathrm{B}_{J} \mathrm{~A}_{J}\right)$ & $\varrho\left(\mathrm{I}-\mathrm{B}_{J} \mathrm{~A}_{J}\right)$ & $\kappa\left(\mathrm{B}_{J} \mathrm{~A}_{J}\right)$ & $\varrho\left(\mathrm{I}-\mathrm{B}_{J} \mathrm{~A}_{J}\right)$ \\
\hline 2 & 10 & 1.36 & 0.19 & 1.36 & 0.19 \\
3 & 22 & 1.71 & 0.26 & 1.72 & 0.27 \\
4 & 79 & 1.97 & 0.36 & 2.11 & 0.42 \\
5 & 312 & 2.08 & 0.41 & 2.39 & 0.54 \\
6 & 1246 & 2.11 & 0.42 & 2.56 & 0.62 \\
7 & 4981 & 2.11 & 0.42 & 2.66 & 0.66 \\
8 & 19921 & 2.12 & 0.42 & 2.73 & 0.69
\end{tabular}

TABLE 4.1. Condition numbers and contraction numbers, when Gauß-Seidel smoother, and $Q_{1}$ elements are used. $\Omega=$ $(-1,1)^{2}, \sigma=3$.

\section{Numerical ReSUlts fOr Poisson equation}

We report numerical results obtained using the finite element library deal. II by W. Bangerth and G. Kanschat $[4,5]$. All computations use meshes with square grid cells. On each cell the finite element space consists of tensor products of polynomials in each coordinate variable of degree at most $d$ (namely $Q_{d}$ ). Multilevel meshes are obtained by a uniform refinement procedure that breaks each coarse cell into four congruent cells.

First we consider the case when $\Omega=(-1,1)^{2}$ and $d=1$. The coarse grid $\mathcal{T}_{1}$ consists of the single cell $\Omega$. In Table 4.1 , we present spectral condition numbers with and without preconditioning. Here we used a block GaußSeidel smoother, where the block partitioning is such that degrees of freedom within an element are grouped together. Estimates for both $\kappa\left(\mathrm{A}_{J}\right)$ and $\kappa\left(\mathrm{B}_{J} \mathrm{~A}_{J}\right)$ (second and third columns in the table) are in accordance with theoretical estimates. The advantage of preconditioning is clear.

Often V-cycle is used as a solver by itself, rather than as a preconditioner. In this case it is important to see if $I-B_{J} A_{J}$ is a contraction independent of the mesh size. (Here I is the identity matrix.) Although we do not have a theoretical result in this direction, our experiments seem to indicate that the spectral radius of $\mathrm{I}-\mathrm{B}_{J} \mathrm{~A}_{J}$, namely $\varrho\left(\mathrm{I}-\mathrm{B}_{J} \mathrm{~A}_{J}\right)$, remains bounded with refinement (see third column of Table 4.1).

The above-mentioned results are obtained with the number of smoothing steps $m(k)=2^{J-k}$, set in accordance with Assumption (3.1). The last two columns of Table 4.1 are included so we can compare these with the case when only one smoothing step before and after coarse grid correction is done $(m(k)=1)$. The former is found to give a slightly better preconditioner.

In Table 4.2, we present results for the variable V-cycle with block Jacobi smoothing. A relaxation parameter of 0.95 (which appeared to be the best computationally) was used. The condition numbers are not as good as the Gauß-Seidel case. However, as the last two columns indicate, with a 


\begin{tabular}{c|cc|cc}
\multirow{2}{*}{$J$} & \multicolumn{2}{|c|}{$m(k)=2^{J-k}$} & \multicolumn{2}{c}{$m(k)=2^{J-k+1}$} \\
\cline { 2 - 5 } & $\kappa\left(\mathrm{B}_{J} \mathrm{~A}_{J}\right)$ & $\varrho\left(\mathrm{I}-\mathrm{B}_{J} \mathrm{~A}_{J}\right)$ & $\kappa\left(\mathrm{B}_{J} \mathrm{~A}_{J}\right)$ & $\varrho\left(\mathrm{I}-\mathrm{B}_{J} \mathrm{~A}_{J}\right)$ \\
\hline 2 & 1.62 & 0.30 & 1.14 & 0.10 \\
3 & 2.23 & 0.40 & 1.36 & 0.17 \\
4 & 2.72 & 0.54 & 1.49 & 0.21 \\
5 & 2.95 & 0.64 & 1.54 & 0.22 \\
6 & 3.02 & 0.67 & 1.56 & 0.22 \\
7 & 3.04 & 0.68 & 1.56 & 0.22
\end{tabular}

TABLE 4.2. Condition numbers and contraction numbers when Jacobi smoother and $Q_{1}$ elements are used. $\Omega=$ $(-1,1)^{2}, \sigma=3$.

\begin{tabular}{c|rcc|rcc} 
& \multicolumn{3}{|c|}{$Q_{2}$ elements $(\sigma=8)$} & \multicolumn{3}{c}{$Q_{3}$ elements $(\sigma=22)$} \\
\cline { 2 - 7 }$J\left(\mathrm{~A}_{J}\right)$ & $\kappa\left(\mathrm{B}_{J} \mathrm{~A}_{J}\right)$ & $\varrho\left(\mathrm{I}-\mathrm{B}_{J} \mathrm{~A}_{J}\right)$ & $\kappa\left(\mathrm{A}_{J}\right)$ & $\kappa\left(\mathrm{B}_{J} \mathrm{~A}_{J}\right)$ & $\varrho\left(\mathrm{I}-\mathrm{B}_{J} \mathrm{~A}_{J}\right)$ \\
\hline 2 & 23 & 2.07 & 0.36 & 77 & 2.97 & 0.53 \\
3 & 69 & 2.11 & 0.39 & 269 & 2.88 & 0.50 \\
4 & 263 & 2.14 & 0.40 & 1061 & 2.90 & 0.52 \\
5 & 1041 & 2.16 & 0.41 & 4235 & 2.92 & 0.52 \\
6 & 4154 & 2.16 & 0.41 & 16934 & 2.92 & 0.52 \\
7 & 16605 & 2.16 & 0.41 & 67731 & 2.92 & 0.52
\end{tabular}

TABLE 4.3. Condition numbers of $\mathrm{A}_{J}$ and $\mathrm{B}_{J} \mathrm{~A}_{J}$ when GaußSeidel smoother and biquadratic $\left(Q_{2}\right)$ and bicubic $\left(Q_{3}\right)$ shape functions are used. $\Omega=(-1,1)^{2}$.

slight increase in number of smoothing steps, the preconditioner with Jacobi smoother also yields good condition numbers.

Table 4.3 shows that the multigrid preconditioner works well for higher order finite element spaces.

We also investigated the variation of extremal eigenvalues of $\mathrm{A}_{J}$ and $\mathrm{B}_{J} \mathrm{~A}_{J}$ with $\sigma$. We found that the minimum eigenvalue of $\mathrm{A}_{J}$ is independent of $\sigma$ as long as the discretization is stable, while its maximum eigenvalue grows linearly with $\sigma$. In contrast the minimum eigenvalue of $\mathrm{B}_{J} \mathrm{~A}_{J}$ decreases with increasing $\sigma$ and its maximum eigenvalue seems bounded with increasing $\sigma$. Figure 1 shows that the condition number of $\mathrm{B}_{J} \mathrm{~A}_{J}$ grows linearly in $\sigma$ for sufficiently large $\sigma$.

In the analysis, we assumed a mild regularity assumption (2.2), that holds for non-convex polygonal domains. We now investigate the performance of the preconditioner on an L-shaped domain $\Omega_{L}=\Omega \backslash[0,1)^{2}$ and a domain with a slit $\Omega_{S}=\Omega \backslash\{0\} \times[0,1)$. While (2.2) holds with $\alpha<2 / 3$ on $\Omega_{L}$, it does not hold for $\Omega_{S}$. We conclude from Table 4.4 that the algorithm yields a good preconditioner in both cases. 


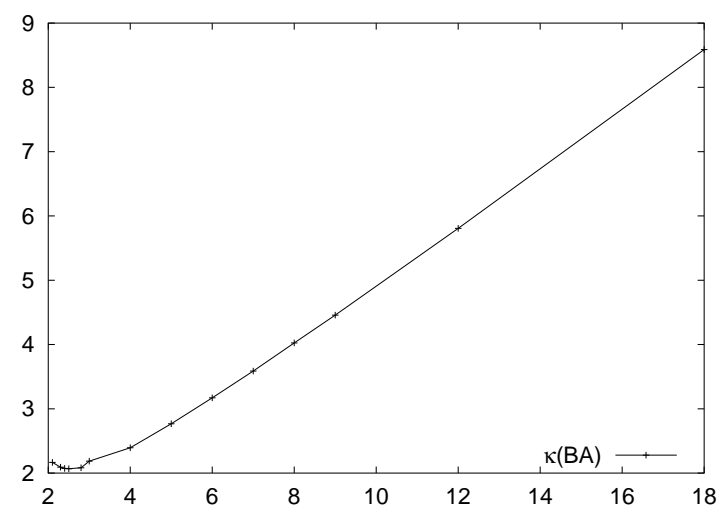

Figure 1. Variation of the condition number of $\mathrm{B}_{6} \mathrm{~A}_{6}$ (ordinate) with $\sigma$ (abscissa).

\begin{tabular}{c|cc|cc}
\multirow{2}{*}{$L$} & \multicolumn{2}{|c|}{$\Omega_{L}$} & \multicolumn{2}{c}{$\Omega_{S}$} \\
\cline { 2 - 5 } & $\kappa\left(\mathrm{B}_{J} \mathrm{~A}_{J}\right)$ & $\varrho\left(\mathrm{I}-\mathrm{B}_{J} \mathrm{~A}_{J}\right)$ & $\kappa\left(\mathrm{B}_{J} \mathrm{~A}_{J}\right)$ & $\varrho\left(\mathrm{I}-\mathrm{B}_{J} \mathrm{~A}_{J}\right)$ \\
\hline 2 & 1.70 & 0.28 & 1.70 & 0.31 \\
3 & 1.96 & 0.38 & 1.92 & 0.35 \\
4 & 2.08 & 0.41 & 2.06 & 0.40 \\
5 & 2.11 & 0.42 & 2.10 & 0.41 \\
6 & 2.11 & 0.42 & 2.11 & 0.42 \\
7 & 2.12 & 0.42 & 2.12 & 0.42
\end{tabular}

TABLE 4.4. Condition numbers and contraction numbers for L-shaped and slit domains using bilinear shape functions and Gauß-Seidel smoothing.

\section{AdVection-Diffusion PROBlems}

The subject of this section is the advection-diffusion problem

$$
\begin{aligned}
-\varepsilon \Delta V+\beta \cdot \nabla V & =f & & \text { on } \Omega, \\
V & =g & & \text { on } \partial \Omega .
\end{aligned}
$$

Here $\varepsilon>0$ is a constant that may be arbitrarily small and $\beta$ is a constant vector (of $O(1)$ magnitude). It is well known that a standard finite element method is inappropriate for this problem [19]. We will introduce a discontinuous Galerkin scheme for this problem, give an error estimate, and report numerical experiments with a multigrid technique that makes good heuristic sense.

The discretization scheme we will consider is obtained by discretizing the term $-\varepsilon \Delta V$ by the interior penalty method, and the transport term $\beta \cdot \nabla V$ by the discontinuous Galerkin method. Let the inflow part of the boundary $\partial \Omega$ be denoted by $\partial \Omega_{-}$, i.e., $\partial \Omega_{-}=\{x \in \partial \Omega: n(x) \cdot \beta<0\}$ (here $n(x)$ is the 
outward normal) and for all $u \in H^{1}\left(\mathcal{T}_{k}\right)$ let

$$
u^{+}(x)=\lim _{\delta \rightarrow 0+} u(x+\delta \beta), \quad u^{-}(x)=\lim _{\delta \rightarrow 0+} u(x-\delta \beta) .
$$

The scheme is motivated by the fact that if the solution $V$ of (5.1) is in $H^{s}(\Omega)$ with $s>3 / 2$, then it satisfies

$$
\varepsilon a_{k}(V, v)+b_{k}(V, v)=F(v) \quad \text { for all } v \in H^{1}\left(\mathcal{T}_{k}\right),
$$

where $H^{1}\left(\mathcal{T}_{k}\right)$ and $a_{k}(\cdot, \cdot)$ are as defined earlier, and

$$
\begin{aligned}
b_{k}(u, v) & =\sum_{\tau \in \mathcal{T}_{k}}(\beta \cdot \nabla u, v)_{\tau}+\sum_{e \in \mathcal{E}_{k}^{0}}\left(u^{+}-u^{-}, v^{+}|n \cdot \beta|\right)_{e}+\sum_{e \in \mathcal{E}_{k}^{-}}\left(u^{+}, v^{+}|n \cdot \beta|\right)_{e}, \\
F(v) & =(f, v)+\sum_{e \in \mathcal{E}_{k}^{\partial}}\left(\frac{\varepsilon \sigma}{\ell_{e}}(g,[v])_{e}-\varepsilon\left(g,\left\langle\partial_{n} v\right\rangle\right)_{e}\right)+\sum_{e \in \mathcal{E}_{k}^{-}}\left(g, v^{+}|n \cdot \beta|\right)_{e} .
\end{aligned}
$$

Here and elsewhere we use the following subsets of $\mathcal{E}_{k}$ :

$$
\begin{array}{ll}
\mathcal{E}_{k}^{0}=\left\{e \in \mathcal{E}_{k}: e \subseteq \Omega\right\}, & \mathcal{E}_{k}^{-}=\left\{e \in \mathcal{E}_{k}: e \subseteq \partial \Omega_{-}\right\}, \\
\mathcal{E}_{k}^{\partial}=\left\{e \in \mathcal{E}_{k}: e \subseteq \partial \Omega\right\}, & \mathcal{E}_{k}^{+}=\left\{e \in \mathcal{E}_{k}: e \subseteq \partial \Omega \backslash \partial \Omega_{-}\right\} .
\end{array}
$$

Our scheme computes an approximation $V_{k} \in M_{k}$ that satisfies

$$
\varepsilon a_{k}\left(V_{k}, v\right)+b_{k}\left(V_{k}, v\right)=F(v) \quad \text { for all } v \in M_{k} .
$$

It can be shown [20] that

$$
\begin{gathered}
b_{k}(u, u)=\frac{1}{2} \sum_{e \in \mathcal{E}_{k}^{-}}\left(u^{+}, u^{+}|n \cdot \beta|\right)_{e}+\frac{1}{2} \sum_{e \in \mathcal{E}_{k}^{+}}\left(u^{-}, u^{-}|n \cdot \beta|\right)_{e} \\
+\frac{1}{2} \sum_{e \in \mathcal{E}_{k}^{0}}\left(u^{+}-u^{-},\left(u^{+}-u^{-}\right)|n \cdot \beta|\right)_{e} .
\end{gathered}
$$

for all $u \in H^{1}\left(\mathcal{T}_{k}\right)$. Thus, $b_{k}(u, u) \geq 0$, and (5.4) has a unique solution. Moreover, the following error estimate holds.

Theorem 5.1. Suppose $V$ and $V_{k}$ solve (5.3) and (5.4) respectively. Assume that $V \in H^{s}(\Omega)$ with $3 / 2<s \leq d+1$. Then in the norm $\|\cdot\|_{\varepsilon, k}$ defined by

$$
\|u\|_{\varepsilon, k}^{2}=\varepsilon a_{k}(u, u)+b_{k}(u, u)+\sum_{\tau \in \mathcal{T}_{k}} h_{k}\|\beta \cdot \nabla u\|_{0, \tau}^{2},
$$

the following error estimate holds:

$$
\left\|V-V_{k}\right\|_{\varepsilon, k} \leq C_{1} \max \left(\sqrt{\varepsilon} h_{k}^{s-1}, h_{k}^{s-1 / 2}\right)|V|_{s, \Omega} .
$$

Here $C_{1}$ is independent of $\varepsilon$ and $h_{k}$.

We give a proof in the next section. Note that in the presence of boundary layers, $|V|_{s, \Omega}$ may be large, and care needs to be taken to design meshes accordingly. Although the theorem is stated for quasiuniform meshes (which we assumed primarily for multigrid analysis), similar estimates on locally 
refined meshes may be obtained. An upcoming work [18] on $h p$-estimates for a different, but somewhat similar method for advection-diffusion problems, seems to have a more exhaustive analysis in this direction. However, our error estimate is in a norm slightly stronger than in [18], due to the final term in (5.6). This term seems to provide more control of gradient of error in the direction of $\beta$ as $\varepsilon$ tends to zero.

Having ensured that the method yields good approximations, we now look for efficient methods for solving the linear system arising from (5.4) for $k=J$. Let $\mathrm{T}_{k}$ be the matrix with its $(i, j)$-th entry equal to $\varepsilon a_{k}\left(\phi_{k}^{j}, \phi_{k}^{i}\right)+$ $b_{k}\left(\phi_{k}^{j}, \phi_{k}^{i}\right)$. The following result shows that the $\mathrm{B}_{J}$ defined by Algorithm 3.1 can be used to precondition $\mathrm{T}_{J}$. For $\mathrm{x}, \mathrm{y} \in \mathbb{R}^{N_{J}}$, we let $[\mathrm{x}, \mathrm{y}]=\mathrm{B}_{J}^{-1} \mathrm{x} \cdot \mathrm{y}$.

Corollary 5.1. The convergence rate of GMRES in the $[\cdot, \cdot]^{1 / 2}$-innerproduct applied to the preconditioned system $\mathrm{B}_{J} \mathrm{\top}_{J} \mathrm{v}_{J}=\mathrm{B}_{J} \mathrm{f}$ is bounded (independently of $\left.\left\{h_{k}\right\}\right)$ by

$$
1-\left(\zeta^{-2} \frac{\varepsilon}{\varepsilon+C|\beta|}\right)^{2} .
$$

Proof. The result essentially follows from the two inequalities

$$
\begin{gathered}
\varepsilon \mathrm{A}_{J} \mathrm{x} \cdot \mathrm{x} \leq \mathrm{T}_{J} \mathrm{x} \cdot \mathrm{x} \text { and } \\
\mathrm{T}_{J} \mathrm{x} \cdot \mathrm{y} \leq(\varepsilon+C|\beta|)\left(\mathrm{A}_{J} \mathrm{x} \cdot \mathrm{x}\right)^{1 / 2}\left(\mathrm{~A}_{J} \mathrm{y} \cdot \mathrm{y}\right)^{1 / 2},
\end{gathered}
$$

for all $\mathrm{x}, \mathrm{y} \in \mathbb{R}^{N_{J}}$. The first inequality is obvious, and the second follows from

$$
\begin{aligned}
b_{J}(u, v) & \leq \sum_{\tau \in \mathcal{T}_{J}}\|\beta \cdot \nabla u\|_{0, \tau}\|v\|_{0, \tau}+\sum_{e \in \mathcal{E}_{J}^{0} \cup \mathcal{E}_{J}^{-}}\left(h_{J}^{-1 / 2}\|[u]\|_{0, e}\right)\left(h_{J}^{1 / 2}\left\|v^{+}|n \cdot \beta|\right\|_{0, e}\right) \\
& \leq C|\beta|\left(\sum_{\tau \in \mathcal{T}_{J}}\|\nabla u\|_{0, \tau}^{2}+\sum_{e \in \mathcal{E}_{J}} \frac{1}{\ell_{e}}\|[u]\|_{0, e}^{2}\right)^{1 / 2}\|v\|_{0, \Omega} \\
& \leq C|\beta| \widetilde{a}_{J}(u, u)^{1 / 2}\|v\|_{0, \Omega} \leq C|\beta| a_{J}(u, u)^{1 / 2} a_{J}(v, v)^{1 / 2}
\end{aligned}
$$

where we have used (2.15) and the Poincaré inequality (2.16). Now, it can easily be shown that the estimate of Theorem 3.1 implies

$$
\zeta^{-1} \mathrm{~B}_{J}^{-1} \mathrm{x} \cdot \mathrm{x} \leq \mathrm{A}_{J} \mathrm{x} \cdot \mathrm{x} \leq \zeta \mathrm{B}_{J}^{-1} \mathrm{x} \cdot \mathrm{x} .
$$

This with (5.7) and (5.8) yields

$$
\begin{gathered}
\varepsilon \zeta^{-1}[\mathrm{x}, \mathrm{x}] \leq\left[\mathrm{B}_{J} \mathrm{\top}_{J} \mathrm{x}, \mathrm{x}\right] \text { and } \\
{\left[\mathrm{B}_{J} \mathrm{\top}_{J} \mathrm{x}, \mathrm{y}\right] \leq(\varepsilon+C|\beta|) \zeta[\mathrm{x}, \mathrm{x}]^{1 / 2}[\mathrm{y}, \mathrm{y}]^{1 / 2} .}
\end{gathered}
$$

Combining this with well-known results in [14] we have the result.

This result gives an efficient method for solving (5.4) whenever $\varepsilon$ is of the same order of magnitude as $\beta$. Indeed, it says that GMRES converges in a fixed number of steps, no matter how highly refined the meshes are. Note that GMRES in the $[\cdot, \cdot]^{1 / 2}$-innerproduct can be realized computationally 
without multiplications by $\mathrm{B}_{J}^{-1}$. (Algorithm 3.1 gives only the action of $\mathrm{B}_{J}$, not $\mathrm{B}_{J}^{-1}$.) Although this gives a good preconditioner when $\varepsilon$ is $O(1)$, the estimate of Corollary 5.1 deteriorates as $\varepsilon$ approaches zero. We will now show how we can arrive at a method that performs independently of $\varepsilon$ as well.

When $\varepsilon=0$, as pointed out in [22], a downwind ordering of mesh elements makes $\mathrm{T}_{k}$ a block triangular matrix. The block partitioning is such that each block corresponds to nodes of one element. Specifically, if $\tau_{1}, \tau_{2}, \ldots \tau_{L}$ is an ordering of elements of $\mathcal{T}_{k}$ such that for any $i$, the inflow part of $\partial \tau_{i}$ is either a subset of $\partial \Omega_{-}$or of the outflow part of $\partial \tau_{j}$ for some $j<i$, then in this ordering $\mathrm{T}_{k}$ is $L \times L$ block triangular. Now we observe that because $T_{k}$ is block triangular, a block Gauß-Seidel iteration in this ordering solves a system involving $\mathrm{T}_{k}$ in one step. If $\varepsilon$ is nonzero but small, $\mathrm{T}_{k}$ is a perturbation of a block triangular matrix, and we can still expect this iteration to work well. Finally, when $\varepsilon$ is $O(1)$, a multigrid cycle with a block Gauß-Seidel smoother (in any ordering) works well.

These observations lead us to modify Algorithm 3.1 by substituting the above-mentioned downwind block Gauß-Seidel matrix (which we denote by $\mathrm{G}_{k}^{\beta}$ ) for $\mathrm{R}_{k}$. Also, since $\mathrm{T}_{k}$ is nonsymmetric anyway, the symmetrizing postsmoothing operation (Step 3 of Algorithm 3.1) may be removed. The resulting preconditioner $\mathrm{B}_{J}^{\beta}$ is given by the following algorithm.

Algorithm 5.1. Set $\mathrm{x}^{0}=0$ and $\mathrm{B}_{1}^{\beta}=\mathrm{A}_{1}^{-1}$. For $k \geq 2$ and any vector $\mathrm{d} \in \mathbb{R}^{N_{k}}$, the vector $\mathrm{B}_{k}^{\beta} \mathrm{d}$ is computed as follows:

(1) Compute $\mathrm{x}^{l}$ for $l=1, \ldots m(k)$ :

$$
\mathrm{x}^{l}=\mathrm{x}^{l-1}+\mathrm{G}_{k}^{\beta}\left(\mathrm{d}-\mathrm{T}_{k} \mathrm{x}^{l-1}\right) .
$$

(2) Set $\mathrm{B}_{k}^{\beta} \mathrm{d}=\mathrm{x}^{m(k)}+\mathrm{C}_{k-1}^{t} \mathrm{~B}_{k-1}^{\beta} \mathrm{C}_{k-1}\left(\mathrm{~d}-\mathrm{T}_{k} \mathrm{x}^{m(k)}\right)$.

We now report a representative numerical experiment using $\mathrm{B}_{J}^{\beta}$, with $m(k)=2^{J-k}$. At the outset, we note that in all our experiments, the computational cost of reordering the unknowns downwind was little, compared with that of the floating point operations involved in solution process. With $\beta=(0.5,0.866)^{t}$, we choose $f$ and $g$ such that

$$
u(x, y)=-\arctan (8(0.5 y-0.866 x))
$$

on the square $\Omega=(-1,1)^{2}$. (This $u(x, y)$ solves (5.1) with $\varepsilon=0$ and $f=0$. There are no boundary layers when $\varepsilon>0$.) We use $Q_{1}$ elements, and solve for $V_{J}$ defined by (5.4). GMRES with $\mathrm{B}_{J}^{\beta}$ as preconditioner and with zero as initial guess is used, and iterations were stopped when residual norm was reduced by $10^{-10}$. The number of iterations required for convergence as a function of $\varepsilon$ and mesh size is reported in Table 5.1. The iteration counts at the upper left corner of the table reflect the fact that the smoother is almost an exact solver. At the lower right part, diffusion dominates, and we recover convergence rates of the elliptic problem. For comparison, we 


\begin{tabular}{r|c|ccccccccc}
\multicolumn{10}{c|}{} & \multicolumn{10}{|c}{$J=2-\log _{2} h_{J}$} \\
& 2 & 3 & 4 & 5 & 6 & 7 & 8 & 9 & 10 \\
\hline$-\infty$ & 1 & 1 & 1 & 1 & 1 & 1 & 1 & 1 & 1 \\
-18 & 2 & 3 & 3 & 3 & 4 & 4 & 5 & 7 & 9 \\
-16 & 3 & 3 & 3 & 4 & 4 & 5 & 7 & 10 & 15 \\
-14 & 3 & 3 & 4 & 4 & 5 & 7 & 10 & 15 & 17 \\
$\log _{2} \varepsilon$ & -12 & 3 & 4 & 5 & 6 & 7 & 10 & 14 & 15 & 14 \\
& -10 & 4 & 5 & 6 & 8 & 11 & 14 & 14 & 14 & 14 \\
& -8 & 5 & 6 & 8 & 11 & 13 & 14 & 15 & 16 & 17 \\
-6 & 6 & 9 & 12 & 14 & 16 & 17 & 18 & 19 & 19 \\
& -4 & 7 & 12 & 15 & 17 & 18 & 19 & 20 & 20 & 20 \\
-2 & 8 & 14 & 17 & 19 & 20 & 21 & 21 & 20 & 20 \\
0 & 9 & 15 & 18 & 21 & 21 & 21 & 21 & 21 & 20 \\
2 & 9 & 15 & 19 & 21 & 21 & 21 & 21 & 21 & 20 \\
$\infty$ & 9 & 15 & 18 & 21 & 21 & 21 & 21 & 21 & 20
\end{tabular}

TABLE 5.1. Iteration counts depending on $\varepsilon$ and refinement level $J$.

have included iteration counts for pure advection problem, i.e., $\varepsilon=0$ (see row labeled $-\infty$ ), and pure elliptic problem, i.e., $\beta=0$ and $\varepsilon=1$ (see row labeled $\infty$ ). Clearly, the iteration counts remain bounded in all the ranges of $\varepsilon$ and refinement levels considered.

\section{Proof of the ERror estimate}

We give a proof of Theorem 5.1. Here the multilevel nature of the spaces and meshes are immaterial. To simplify notation, let us define

$$
\langle u, v\rangle_{S}=\sum_{e \in S}(u, v|n \cdot \beta|)_{e}
$$

where $S$ is any of the sets $\varepsilon_{k}^{0}, \mathcal{E}_{k}^{-}, \mathcal{E}_{k}^{\partial}$, or $\varepsilon_{k}^{+}$. Also, for $u \in H^{1}\left(\mathcal{T}_{k}\right)$, let $u_{\beta} \in L^{2}(\Omega)$ be defined by

$$
\left.u_{\beta}\right|_{\tau}=\beta \cdot \nabla u \quad \text { for all } \tau \in \mathcal{T}_{k} .
$$

We begin with a stability result, which can also be thought of as an inf-sup condition.

Lemma 6.1. There exists a $C_{2}>0$ such that for all $u \in M_{k}$,

$$
C\|u\|_{\varepsilon, k}^{2} \leq \varepsilon a_{k}\left(u, C_{2} u+h_{k} u_{\beta}\right)+b_{k}\left(u, C_{2} u+h_{k} u_{\beta}\right) .
$$

Proof. We will prove that for any $C_{2}$, there exist constants $c_{2}^{\prime}, c_{2}^{\prime \prime}>0$ such that

$$
\begin{aligned}
& b_{k}\left(u, C_{2} u+h_{k} u_{\beta}\right) \geq C_{2} b_{k}(u, u)-c_{2}^{\prime} b_{k}(u, u)+h_{k}\left\|u_{\beta}\right\|_{0, \Omega}^{2} / 2, \\
& a_{k}\left(u, C_{2} u+h_{k} u_{\beta}\right) \geq C_{2} a_{k}(u, u)-c_{2}^{\prime \prime} a_{k}(u, u)
\end{aligned}
$$


for any $u \in M_{k}$. This is sufficient, because we can choose $C_{2}=1+$ $\max \left(c_{2}^{\prime}, c_{2}^{\prime \prime}\right)$ in (6.1) and (6.2), and complete the proof.

To prove $(6.1)$, we start with

$$
\begin{aligned}
b_{k}\left(u, C_{2} u+h_{k} u_{\beta}\right)= & C_{2} b_{k}(u, u)+h_{k}\left\|u_{\beta}\right\|_{0, \Omega}^{2} \\
& +h_{k}\left\langle u^{+}-u^{-},\left(u_{\beta}\right)^{+}\right\rangle_{\mathcal{E}_{k}^{0}}+h_{k}\left\langle u^{+},\left(u_{\beta}\right)^{+}\right\rangle_{\mathcal{E}_{k}^{-}},
\end{aligned}
$$

and apply Cauchy-Schwarz inequality, a trace theorem, and a scaling argument. Then, for any $\delta>0$,

$$
\begin{aligned}
b_{k}\left(u, C_{2} u+h_{k} u_{\beta}\right) \geq & C_{2} b_{k}(u, u)+h_{k}\left\|u_{\beta}\right\|_{0, \Omega}^{2}-\delta C h_{k}\left\|u_{\beta}\right\|_{0, \Omega}^{2} \\
& -\frac{1}{2 \delta}\left(\left\langle u^{+}-u^{-}, u^{+}-u^{-}\right\rangle_{\mathcal{E}_{k}^{0}}+\left\langle u^{+}, u^{+}\right\rangle_{\mathcal{E}_{k}^{-}}\right) .
\end{aligned}
$$

Choosing $\delta$ appropriately, and using (5.5), we get (6.1).

To prove (6.2), it suffices to prove that there is $c_{2}^{\prime \prime}$ such that

$$
\left|a_{k}\left(u, h_{k} u_{\beta}\right)\right| \leq c_{2}^{\prime \prime} a_{k}(u, u) .
$$

This requires bounding the four sums on the right hand side of

$$
\begin{aligned}
a_{k}\left(u, h_{k} u_{\beta}\right)= & \sum_{\tau \in \mathcal{T}_{k}}\left(\nabla u, h_{k} \nabla u_{\beta}\right)_{\tau}+ \\
& \sum_{e \in \mathcal{E}_{k}}\left(\frac{\sigma}{\ell_{e}}\left([u],\left[h_{k} u_{\beta}\right]\right)_{e}-\left(\left\langle\partial_{n} u\right\rangle,\left[h_{k} u_{\beta}\right]\right)_{e}-\left([u], h_{k}\left\langle\partial_{n} u_{\beta}\right\rangle\right)_{e}\right),
\end{aligned}
$$

by $a_{k}(u, u)$. It can be seen that such a bound does hold for each sum by using the inverse inequalities (2.6) and (2.13), the trace inequality (2.12), and (2.15). For example, consider the last sum:

$$
\begin{aligned}
\sum_{e \in \mathcal{E}_{k}}\left([u], h_{k}\left\langle\partial_{n} u_{\beta}\right\rangle\right)_{e} & \leq\left(\sum_{e \in \mathcal{E}_{k}} h_{k}^{-1}\|[u]\|_{0, e}^{2}\right)^{1 / 2}\left(\sum_{\tau \in \mathcal{T}_{k}} h_{k}^{2}\left\|\nabla u_{\beta}\right\|_{0, \tau}^{2}\right)^{1 / 2} \\
& \leq C \widetilde{a}_{k}(u, u)^{1 / 2}\left\|u_{\beta}\right\|_{0, \Omega} \leq C a_{k}(u, u)^{1 / 2}\left\|u_{\beta}\right\|_{0, \Omega} .
\end{aligned}
$$

Proof of Theorem 5.1. Let $\widetilde{V}_{k}$ be an interpolant [26] of $V$ that is continuous on $\Omega$ and satisfies the approximation property

$$
\left\|V-\widetilde{V}_{k}\right\|_{0, \Omega}+h_{k}\left|V-\widetilde{V}_{k}\right|_{1, \Omega} \leq C h_{k}^{s}|V|_{s, \Omega} .
$$

Using a trace inequality, it is easy to see that this implies that

$$
\left\|V-\widetilde{V}_{k}\right\|_{\varepsilon, k} \leq C\left(\sqrt{\varepsilon} h^{s-1}+h^{s-1 / 2}\right)|V|_{s, \Omega} .
$$

Now, if we let $E_{k}=V_{k}-\widetilde{V}_{k}$, then

$$
\left\|V-V_{k}\right\|_{\varepsilon, k} \leq\left\|V-\widetilde{V}_{k}\right\|_{\varepsilon, k}+\left\|E_{k}\right\|_{\varepsilon, k} .
$$

Thus, it suffices to establish a bound for $E_{k}$ similar to that in (6.5). 
Because of the consistency of the method as given by (5.3), we have

$$
\varepsilon a_{k}\left(V-V_{k}, w_{k}\right)+b_{k}\left(V-V_{k}, w_{k}\right)=0 \quad \text { for all } w_{k} \in M_{k} .
$$

Therefore, denoting $E_{k}^{\beta}=C_{2} E_{k}+h_{k}\left(E_{k}\right)_{\beta}$, we have by Lemma 6.1,

$$
\begin{aligned}
C\left\|E_{k}\right\|_{\varepsilon, k}^{2} & \leq \varepsilon a_{k}\left(E_{k}, E_{k}^{\beta}\right)+b_{k}\left(E_{k}, E_{k}^{\beta}\right) \\
& =\varepsilon a_{k}\left(V-\widetilde{V}_{k}, E_{k}^{\beta}\right)+b_{k}\left(V-\widetilde{V}_{k}, E_{k}^{\beta}\right) .
\end{aligned}
$$

Now we will use continuity properties of $a_{k}(\cdot, \cdot)$ and $b_{k}(\cdot, \cdot)$ to estimate the quantities on the right hand side above.

To estimate the first term on the right hand side of (6.7), we first use Cauchy-Schwarz inequality:

$$
\begin{aligned}
& a_{k}\left(V-\widetilde{V}_{k}, E_{k}^{\beta}\right) \leq\left|V-\widetilde{V}_{k}\right|_{1, \Omega}\left(\sum_{\tau \in \mathcal{T}_{k}}\left\|\nabla E_{k}^{\beta}\right\|_{0, \tau}^{2}\right)^{1 / 2}+ \\
& \left(\sum_{e \in \mathcal{E}_{k}} h_{k}\left\|\left\langle\partial_{n}\left(V-\widetilde{V}_{k}\right)\right\rangle\right\|_{0, e}^{2}+\sum_{e \in \mathcal{E}_{k}^{\partial}} h_{k}^{-1}\left\|V-\widetilde{V}_{k}\right\|_{0, e}^{2}\right)^{1 / 2}\left(\sum_{e \in \mathcal{E}_{k}} h_{k}^{-1}\left\|\left[E_{k}^{\beta}\right]\right\|_{0, e}^{2}\right)^{1 / 2} .
\end{aligned}
$$

Now, since the interpolant preserves polynomials of degree $d \geq 1$, a BrambleHilbert argument (for fractional order spaces) readily shows that

$$
h_{k} \sum_{e \in \mathcal{E}_{k}}\left\|\left\langle\partial_{n}\left(V-\widetilde{V}_{k}\right)\right\rangle\right\|_{0, e}^{2} \leq h_{k}^{2 s-2}|V|_{s, \Omega}^{2} .
$$

Local inverse inequalities imply

$$
\begin{gathered}
\sum_{\tau \in \mathcal{T}_{k}}\left\|\nabla E_{k}^{\beta}\right\|_{0, \tau}^{2} \leq C \sum_{\tau \in \mathcal{T}_{k}}\left(\left\|\nabla E_{k}\right\|_{0, \tau}^{2}+\left\|\left(E_{k}\right)_{\beta}\right\|_{0, \tau}^{2}\right), \quad \text { and } \\
\sum_{e \in \mathcal{E}_{k}} h_{k}^{-1}\left\|\left[E_{k}^{\beta}\right]\right\|_{0, e}^{2} \leq C \sum_{e \in \mathcal{E}_{k}} h_{k}^{-1}\left\|\left[E_{k}\right]\right\|_{0, e}^{2}+C \sum_{\tau \in \mathcal{T}_{k}}\left\|\left(E_{k}\right)_{\beta}\right\|_{0, \tau}^{2} .
\end{gathered}
$$

These estimates together with a trace theorem, (6.4), and (2.15), yield

$$
\varepsilon a_{k}\left(V-\widetilde{V}_{k}, E_{k}^{\beta}\right) \leq C \varepsilon h_{k}^{s-1}|V|_{s, \Omega} a_{k}\left(E_{k}, E_{k}\right)^{1 / 2} .
$$

Now we estimate the second term on the right hand side of (6.7). It can be seen using the integration by parts formula

$$
b_{k}(u, v)=\left(u,-v_{\beta}\right)+\left\langle u^{-}, v^{-}-v^{+}\right\rangle_{\mathcal{E}_{k}^{0}}+\left\langle u^{-}, v^{-}\right\rangle_{\mathcal{E}_{k}^{+}},
$$

that for any $u \in H^{1}\left(\mathcal{T}_{k}\right)$ and $v \in M_{k}$,

$$
\begin{aligned}
b_{k}\left(u, C_{2} v+h_{k} v_{\beta}\right) \leq C\left(h_{k}^{-1 / 2}\|u\|_{0, \Omega}+\left\langle u^{-}, u^{-}\right\rangle_{\mathcal{E}_{k}^{0}}^{1 / 2}\right. & \\
& \left.+\left(\sum_{\tau \in \mathcal{T}_{k}} h_{k}\left\|u_{\beta}\right\|^{2}\right)^{1 / 2}+b_{k}(u, u)^{1 / 2}\right)\|v\|_{\varepsilon, k} .
\end{aligned}
$$


Using this with $u=V-\widetilde{V}_{k}$ and $v=E_{k}$, a trace inequality, and (6.4), we get that

$$
b_{k}\left(V-\widetilde{V}_{k}, E_{k}^{\beta}\right) \leq C h_{k}^{s-1 / 2}|V|_{s, \Omega}\left\|E_{k}\right\|_{\varepsilon, k} .
$$

Combining (6.8) and (6.9) and using it in (6.7), we have that

$$
\left\|E_{k}\right\|_{\varepsilon, k} \leq C\left(\sqrt{\varepsilon} h_{k}^{s-1}+h_{k}^{s-1 / 2}\right)|V|_{s, \Omega} .
$$

This together with (6.5) proves the theorem.

\section{Conclusion}

We have presented multigrid techniques for two DG schemes: one for elliptic problems and another for singularly perturbed advection-diffusion problems. For the latter DG scheme we have also proved an error estimate.

In the former case, our analysis predicts convergence rates of the multigrid method independent of the mesh size, and numerical experiments bear this out. It is clear from the analysis that second order elliptic boundary value problems more general than (2.1) can be considered. Although our main theorem was for the V-cycle, results for W-cycle with "sufficiently many" smoothings can also be obtained $[6,16]$. In practical computations, a Vcycle with only one smoothing at all levels may be adequate, if it is used as a preconditioner. Our numerical experiments in Section 3 support this.

For the advection-diffusion equation (5.1), we presented a method that behaves uniformly in diffusion dominated as well as convection dominated regime. Various extensions to what we have presented are possible. Inclusion of an $L^{2}$ term in (5.1) presents little difficulty. One can also consider a spatially dependent nonnegative coefficient $\varepsilon$ using the framework introduced in [18]. When $\beta$ is not constant, Theorem 5.1 would require modification. In this case, the multigrid technique presented still applies, but downwind sorting algorithms become more complicated. Ideas in [17] may be of help here.

Acknowledgements. The authors are grateful to Bernardo Cockburn and Dominik Schötzau for helpful discussions.

\section{REFERENCES}

[1] D. Arnold, F. Brezzi, B. Cockburn, and D. Marini. Discontinuous Galerkin methods for elliptic problems. In B. Cockburn, G. Karniadakis, and C.-W. Shu, editors, First International Symposium on Discontinuous Galerkin Methods, volume 11 of Lecture Notes in Computational Science and Engineering, pages 89-101. Springer Verlag, February 2000.

[2] D. N. Arnold. An interior penalty finite element method with discontinuous elements. SIAM J. Numer. Anal., 19(4):742-760, 1982.

[3] I. Babuška. The finite element method with penalty. Math. Comp., 27:221-228, 1973.

[4] W. Bangerth and G. Kanschat. Concepts for object-oriented finite element software the deal. II library. Preprint 99-43, SFB 359, Universität Heidelberg, October 1999. 
[5] W. Bangerth and G. Kanschat. deal.II Differential Equations Analysis Library, Technical Reference, October 1999. http://gaia.iwr.uni-heidelberg.de/ deal/.

[6] J. H. Bramble. Multigrid Methods. Number 294 in Pitman research notes in mathematics series. Longman Scientific \& Technical, Harlow, UK, 1993.

[7] J. H. Bramble and J. E. Pasciak. The analysis of smoothers for multigrid algorithms. Math. Comp., 58(198):467-488, April 1992.

[8] J. H. Bramble, J. E. Pasciak, and J. Xu. The analysis of multigrid algorithms with nonnested spaces or noninherited quadratic forms. Math. Comp., 56(193):1-34, January 1991.

[9] A. Brandt. Multigrid techniques: 1984 guide with applications to fluid dynamics. Gesellschaft für Mathematik und Datenverarbeitung m.b.H., Bonn, St. Augustin, 1984.

[10] F. Brezzi and M. Fortin. Mixed and Hybrid Finite Element Methods. Number 15 in Springer Series in Computational Mathematics. Springer-Verlag, New York, 1991.

[11] P. Castillo, B. Cockburn, I. Perugia, and D. Schötzau. An a priori error analysis of the LDG method for elliptic problems. SIAM J. Numer. Anal., 2000. To appear.

[12] B. Cockburn. Discontinuous Galerkin methods for convection-dominated problems. In High-order methods for computational physics, pages 69-224. Springer, Berlin, 1999.

[13] J. Douglas, Jr. and T. Dupont. Interior penalty procedures for elliptic and parabolic Galerkin methods. In R. Glowinski and J. L. Lions., editors, Computing methods in applied sciences, pages 207-216. Lecture Notes in Phys., Vol. 58, Berlin, 1976. Springer. Second International Symposium on Computing Methods in Applied Sciences and Engineering, held at Versailles, Dec. 1975.

[14] S. C. Eisenstat, H. C. Elman, and M. H. Schultz. Variational iterative methods for nonsymmetric systems of linear equations. SIAM J. Numer. Anal., 20(2):345-357, April 1983.

[15] P. Grisvard. Elliptic Problems in Nonsmooth Domains. Number 24 in Monographs and Studies in Mathematics. Pitman Advanced Publishing Program, Marshfield, Massachusetts, 1985.

[16] W. Hackbusch. Multi-Grid Methods and Applications. Number 4 in Springer series in Computational Mathematics. Springer-Verlag, Berlin, 1985.

[17] W. Hackbusch and T. Probst. Downwind Gauss-Seidel smoothing for convection dominated problems. Numer. Linear Algebra Appl., 4(2):85-102, 1997.

[18] P. Houston, C. Schwab, and E. Süli. Discontinuous hp-finite element methods for advection-diffusion problems. Technical Report 00/15, Oxford University Computing Lab, 2000.

[19] C. Johnson. Numerical solution of partial differential equations by the finite element method. Cambridge University Press, Cambridge, 1995.

[20] C. Johnson and J. Pitkäranta. An analysis of the discontinuous Galerkin method for a scalar hyperbolic equation. Math. Comp., 46(173):1-26, 1986.

[21] R. B. Kellog. Interpolation between subspaces of a Hilbert space. Technical Note BN-719, University of Maryland, College Park, Maryland, November 1971.

[22] P. Lasaint and P.-A. Raviart. On a finite element method for solving the neutron transport equation. In C. de Boor, editor, Mathematical aspects of finite elements in partial differential equations, pages 89-123. Academic Press, New York, 1974. Proceedings of a symposium conducted by Math. Res. Center, Univ. of WisconsinMadison, in April, 1974.

[23] I. Persson, K. Samuelsson, and A. Szepessy. On the convergence of multigrid methods for flow problems. Electron. Trans. Numer. Anal., 8:46-87 (electronic), 1999.

[24] C. Pflaum. Robust convergence of multilevel algorithms for convection-diffusion equations. SIAM J. Numer. Anal., 37(2):443-469 (electronic), 2000. 
[25] T. Rusten, P. S. Vassilevski, and R. Winther. Interior penalty preconditioners for mixed finite element approximations of elliptic problems. Math . Comp., 65(214):447466, April 1996.

[26] L. R. Scott and S. Zhang. Finite element interpolation of nonsmooth functions satisfying boundary conditions. Math. Comp., 54(190):483-493, April 1990.

[27] M. F. Wheeler. An elliptic collocation-finite element method with interior penalties. SIAM J. Numer. Anal., 15(1):152-161, 1978.

Institute for Mathematics and its Applications, University of Minnesota, MinneAPOLIS, MN 55455.

E-mail address: jayg@ima.umn.edu

Institut für Angewandte Mathematik, Universität Heidelberg, INF 293/294, D-69121 Heidelberg.

E-mail address: guido.kanschat@na-net.ornl.gov 